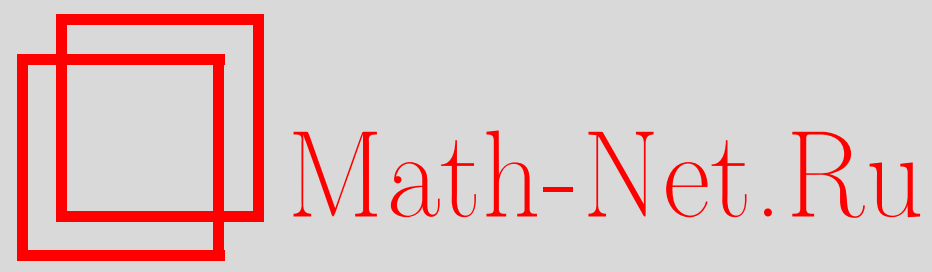

А. Н. Тимашёв, Асимптотические разложения для распределения числа компонент в случайных отображениях и разбиениях, Дискрет. матем., 2011, том 23, выпуск 2, 66-75

DOI: https://doi.org/10.4213/dm1142

Использование Общероссийского математического портала Math-Net.Ru подразумевает, что вы прочитали и согласны с пользовательским соглашением http://www.mathnet.ru/rus/agreement

Параметры загрузки:

IP : 18.208 .226 .222

26 апреля 2023 г., 12:05:37 


\title{
Асимптотические разложения для распределения числа компонент в случайных отображениях и разбиениях
}

\author{
(C) 2011 г. .Н.Тимашёв
}

\begin{abstract}
Рассматривается класс всех $n^{n}$ однозначных отображений $n$-элементного множества в себя. В предположении, что все такие отображения имеют одинаковые вероятности, равные $n^{-n}$, изучается распределение случайной величины $v_{n}$, равной числу компонент связности в случайно выбранном отображении. Выведены асимптотические оценки вероятности $\mathbf{P}\left\{v_{n}=N\right\}$ в предположении, что $n, N \rightarrow \infty$ так, что отношение $N / \ln n$ отграничено от нуля и бесконечности. В частном случае, когда $n, N \rightarrow \infty$ так, что $N=\frac{1}{2} \ln n+o(\ln n)$, для этой вероятности получено полное асимптотическое разложение.

Аналогичное асимптотическое разложение выведено для $\mathbf{P}\left\{\xi_{n}=M\right\}$, где $\xi_{n}-$ случайная величина, равная числу циклов в подстановке, выбираемой случайно равновероятно из множества всех $n$ ! подстановок степени $n$, а также для вероятности $\mathbf{P}\left\{\theta_{n}=N\right\}$, где $\theta_{n}$ - число блоков в случайном равновероятном разбиении $n$-элементного множества.
\end{abstract}

Рассмотрим класс всех $n^{n}$ однозначных отображений $n$-элементного множества в себя. Задавая на этом классе равновероятностное распределение, будем рассматривать случайную величину $v^{n}$, равную числу компонент связности в случайно выбранном отображении. Известно (см., например, [1]), что при $N=1, \ldots, n$

$$
\mathbf{P}\left\{v_{n}=N\right\}=\frac{n !}{N ! n^{n}} \frac{1}{2 \pi i} \int \frac{\left(-\ln (1-\theta(z))^{N}\right.}{z^{n+1}} d z,
$$

где

$$
\theta(z)=\sum_{k=1}^{\infty} \frac{k^{k-1}}{k !} z^{k}, \quad|z| \leqslant e^{-1},
$$

и интегрирование в (1) осуществляется по окружности $|z|=z_{0} \in\left(0, e^{-1}\right)$, пробегаемой в положительном направлении.

Известно также (см. стр. 85 в [2]), что если $n, N$ стремятся к бесконечности так, что

$$
N=\frac{1}{2} \ln n+x \sqrt{\frac{1}{2} \ln n}, \quad|x| \leqslant C,
$$


где $C-$ постоянная, то

$$
\mathbf{P}\left\{v_{n}=N\right\}=\frac{1}{\sqrt{\pi \ln n}} e^{-x^{2} / 2}(1+o(1))
$$

равномерно относительно $x \in[-C, C]$.

Из оценки (4) следует, что при условии (3) случайная величина $\sqrt{2 / \ln n}\left(v_{n}-\frac{1}{2} \ln n\right)$ имеет в пределе стандартное нормальное распределение [3].

Оценки вероятности $\mathbf{P}\left\{v_{n}=N\right\}$ для случая, когда $N=N(n)$ при $n \rightarrow \infty$ меняется так, что $N \ln N=o(\ln n)$, можно найти в [4]. В предположении, что $n, N \rightarrow \infty$ так, что

$$
1<\beta_{0} \leqslant \beta=n N^{-1} \leqslant \beta_{1}<\infty,
$$

где $\beta_{0}, \beta_{1}-$ постоянные, а также при условии, что разность $n-N=o(\sqrt{n})$ и неотрицательна, такого рода оценки получены в [1].

Случай, когда $\ln n=o(N)$ and $N=O\left((\ln n)^{2}\right)$ при $n \rightarrow \infty$, рассматривался в [5].

Используя соотношения (1), (2) и применяя метод перевала, в этой работе мы доказываем следующие утверждения.

Теорема 1. Пусть $n, N \rightarrow \infty$ так, что

$$
0<\gamma_{0} \leqslant \gamma=\frac{N}{\ln n} \leqslant \gamma_{1}
$$

где $\gamma_{0} и \gamma_{1}-$ постоянные. Тогда равномерно относительно $\gamma \in\left[\gamma_{0}, \gamma_{1}\right]$

$$
\mathbf{P}\left\{v_{n}=N\right\}=\sqrt{\frac{2 \gamma}{n}} \exp (\varphi(\gamma)) \frac{1}{N !}\left(\frac{1}{2} \ln n\right)^{N}(1+o(1))
$$

где

$$
\varphi(\gamma)=\gamma(1-\ln (2 \gamma))-\frac{1}{2}<0
$$

при $\gamma>0, \gamma \neq 1 / 2, \varphi(1 / 2)=0$.

Следствие 1. При условиях теоремы 1

$$
\mathbf{P}\left\{v_{n}=N\right\}=\frac{\exp (\varphi(\gamma))}{\sqrt{\pi \ln n}} n^{\varphi(\gamma)}(1+o(1))
$$

равномерно относительно $\gamma \in\left[\gamma_{0}, \gamma_{1}\right]$.

Теорема 2. Пусть $n, N \rightarrow N$ так, что

$$
N=\frac{1}{2} \ln n+x \sqrt{\frac{1}{2} \ln n}
$$

где

$$
x=x(n)=o(\sqrt{\ln n}),
$$

тогда

$$
\mathbf{P}\left\{v_{n}=N\right\}=\frac{1}{\sqrt{\pi \ln n}} \exp \left(-\frac{x^{2}}{2}+\sum_{m=1}^{\infty} \frac{(-1)^{m-1} x^{m+2}}{(m+1)(m+2)}\left(\frac{2}{\ln n}\right)^{m / 2}\right)(1+o(1)) .
$$


Ряд в (11) сходится при всех достаточно больших $n$ и является асимптотическим разложением в том смысле, что если при $n \rightarrow \infty$

$$
x=x(n)=o\left((\ln n)^{(M+1) /(2 M=6)}\right),
$$

mo

$$
\mathbf{P}\left\{v_{n}=N\right\}=\frac{1}{\sqrt{\pi \ln n}} \exp \left(-\frac{x^{2}}{2}+\sum_{m=1}^{M} \frac{(-1)^{m-1} x^{m+2}}{(m+1)(m+2)}\left(\frac{2}{\ln n}\right)^{m / 2}\right)(1+o(1)),
$$

где $M=0,1, \ldots$

Следствие 2. Если $x=x(n)$ при $n \rightarrow \infty$ меняется так, что

$$
x(n)=o\left((\ln n)^{1 / 6}\right),
$$

то оценка (4) остается справедливой.

Следствие 3. Если $n \rightarrow \infty$ and $x=x(n)=o(\ln n)^{1 / 4}$, mo

$$
\mathbf{P}\left\{v_{n}=N\right\}=\frac{1}{\sqrt{\pi \ln n}} \exp \left(-\frac{x^{2}}{2}+\frac{x^{3}}{6} \sqrt{\frac{2}{\ln n}}\right)(1+o(1)) .
$$

Доказательство теоремы 1. При $0<|z|<e^{-1}$ и условиях (5) положим

$$
\begin{aligned}
& \omega(z)=\frac{\gamma \ln n}{n} \ln (A(z))-\ln z, \\
& A(z)=-\ln (1-\theta(z)),
\end{aligned}
$$

где функция $\theta(z)$ определена в (2). Тогда, согласно (1),

$$
\mathbf{P}\left\{v_{n}=N\right\}=\frac{n !}{N ! n^{n} 2 \pi i} \int \frac{\exp (n \omega(z)}{z} d z, \quad N=1, \ldots, n .
$$

Интегрирование в (16), как и в (1), осуществляется по окружности $|z|=z_{0} \in\left(0, e^{-1}\right)$, пробегаемой в положительном направлении. Если положить $z_{0}=t_{0} e^{-t_{0}}$, где $t=t_{0} \in$ $(0,1)$ - единственный корень уравнения

$$
-t^{-1}(1-t)^{2} \ln (1-t)=\frac{\gamma \ln n}{n}
$$

то, согласно известному свойству функции $\theta(z)$, выполняется равенство $t_{0}=\theta\left(z_{0}\right)$, и, поскольку

$$
z(\theta)^{\prime}(z)=\frac{\theta(z)}{1-\theta(z)}
$$

при $0<|z|<e^{-1}$, согласно (14), (15), (17) $\omega^{\prime}\left(z_{0}\right)=0$.

При $n \rightarrow \infty$ из (5), (17) следует, что

$$
\begin{aligned}
z_{0} & \rightarrow e^{-1}, \\
t_{0}=\theta\left(z_{0}\right) & \rightarrow \theta\left(e^{-1}\right)=1 .
\end{aligned}
$$


В [1] показано, что при таких условиях (с учетом (17))

$$
\begin{aligned}
\exp \left(A\left(z_{0}\right)\right)=\left(1-\theta\left(z_{0}\right)\right) & =\sqrt{\frac{n}{2 \gamma \ln n} \ln \left(\frac{n}{\gamma \ln n}\right)}(1+o(1)) \\
& =\sqrt{\frac{n}{2 \gamma}}(1+o(1)) .
\end{aligned}
$$

Из (18) следует, что

$$
A\left(z_{0}\right)=\frac{1}{2} \ln n-\frac{1}{2} \ln (2 \gamma)+o(1) .
$$

Используя (17), оценку (19) можно уточнить. Полагая

$$
y=1-t_{0} \in(0,1),
$$

представим уравнение (17) в виде

$$
-y^{2} \ln y(1-y)^{-1}=\frac{\gamma \ln n}{n} .
$$

Если $n \rightarrow \infty$, то $y \rightarrow 0$ и

$$
y^{2}(1-y)^{-1}=\sum_{m=2}^{\infty} y^{m}
$$

Подставляя (21) в (20) и используя стандартную технику асимптотических оценок, после элементарных преобразований получаем, что

$$
\begin{aligned}
y & =\sqrt{\frac{2 \gamma}{n}}\left(1+\frac{\ln (2 \gamma)}{2 \ln n}+o(1 / \ln n)\right) \\
A\left(z_{0}\right) & =-\ln y=\frac{1}{2} \ln n-\frac{1}{2} \ln (2 \gamma)-\frac{\ln (2 \gamma)}{2 \ln n}+o(1 / \ln n), \\
\left(A\left(z_{0}\right)\right)^{N} & =\left(\frac{1}{2} \ln n\right)^{N}+\exp (-\gamma \ln (2 \gamma))(1+o(1)) .
\end{aligned}
$$

Кроме того, из равенства

$$
z_{0}=(1-y) e^{y-1}
$$

следует, что

$$
z_{0}^{-(n+1)}=\exp (n+1+\gamma)(1+O(1 / \ln n))
$$

Таким образом, согласно (22), (23)

$$
\frac{\left(A\left(z_{0}\right)\right)^{N}}{z_{0}^{n+1}}=\left(\frac{1}{2} \ln n\right)^{N} \exp (n+1+\gamma(1-\ln (2 \gamma)))(1+O(1 / \ln n)) .
$$

Используя (14), (15), (17), а также оценку

$$
-\ln \left(1-t_{0}\right)=\frac{1}{2} \ln n(1+O(1 / \ln n)),
$$


нетрудно проверить, что

$$
\begin{aligned}
\omega^{\prime \prime}\left(z_{0}\right) & =\frac{\gamma \ln n}{n}\left(-\frac{t_{0}^{2}\left(3-t_{0}\right)}{z_{0}^{2}\left(1-t_{0}\right)^{4} \ln \left(1-t_{0}\right)}-\frac{n^{2}}{\gamma^{2}(\ln n)^{2} z_{0}^{2}}\right)+\frac{1}{z_{0}^{2}} \\
& =\frac{n e^{2}}{\gamma}(1+O(1 / \ln n)) .
\end{aligned}
$$

Из (25) в частности следует, что $\omega^{\prime \prime}\left(z_{0}\right)>0$ при всех достаточно больших значениях $n$. Таким образом, $z_{0}-$ точка перевала первого порядка функции $\omega(z)$.

Следствием (24) и (25) является соотношение

$$
\frac{\left(A\left(z_{0}\right)\right)^{N}}{z_{0}^{n+1} \sqrt{\pi e n \omega^{\prime \prime}\left(z_{0}\right)}}=\left(\frac{1}{2} \ln n\right)^{N} \frac{1}{n} \sqrt{\frac{\gamma}{\pi}} \exp \left(n+\gamma(1-\ln (2 \gamma))^{1 / 2}\right)(1+O(1 / \ln n)) .
$$

Используя стандартную технику метода перевала, получаем, что при $n \rightarrow \infty$

$$
\frac{1}{2 \pi i} \int_{|z|=z_{0}} \frac{1}{z} \exp (n \omega(z)) d z=\frac{\left(A\left(z_{0}\right)\right)^{N}}{z_{0}^{n+1} \sqrt{\pi e n \omega^{\prime \prime}\left(z_{0}\right)}}(1+o(1)) .
$$

Оценка (6) следует из формул (7), (16), (26), (27) и формулы Стирлинга для $n$ !. Теорема 1 доказана.

Оценка (8) следует из (5), (6) и формулы Стирлинга для $N$ !. Следствие 1 установлено.

Проиллюстрируем оценку (6) на примере, когда $n=20000$ и $N=10$. При таких условиях оценка (6) дает значение 0,02009 (с точностью до $10^{-5}$ ). В то же время оценка работы [5] дает значение 0,021150 (с той же точностью), а использование локальной нормальной теоремы для случайной величины $v_{n}$ (см. оценку (4)) дает значение 0,01368 (по-видимому, менее точное по сравнению с двумя предыдущими).

Доказательство теоремы 2. Для обоснования оценки (11) при условиях (9), (10) воспользуемся формулой (8). При таких условиях

$$
\gamma=\frac{N}{\ln n}=\frac{1}{2}+\frac{x}{\sqrt{2 \ln n}}=\frac{1}{2}+o(1),
$$

и, согласно (7),

$$
\varphi(\gamma)=o(1), \quad \exp (\varphi(\gamma))=1+o(1)
$$

Далее,

$$
\begin{aligned}
n^{\varphi(\gamma)} & =\exp (\varphi(\gamma) \ln n), \\
\varphi(1 / 2) & =\varphi^{\prime}(1 / 2)=0, \\
\frac{1}{k !} \varphi^{(k)}(1 / 2) & =(-1)^{k-1} \frac{2^{k-1}}{k(k-1)},
\end{aligned}
$$

где $k=2,3, \ldots$ При этом, если $0<\gamma<1$, то

$$
\varphi(\gamma)=-(\gamma-1 / 2)^{2}+\sum_{k=3}^{\infty}(-1)^{k-1} \frac{2^{k-1}}{k(k-1)}(\gamma-1 / 2)^{k},
$$


и ряд в (30) сходится.

При наших условиях

$$
\gamma-\frac{1}{2}=\frac{x}{\sqrt{2 \ln n}},
$$

поэтому, согласно (30), при $m=k-2$ получаем, что

$$
\varphi(\gamma) \ln n=-\frac{x^{2}}{2}+\sum_{m=1}^{\infty} \frac{(-1)^{m-1} x^{m+2}}{(m+1)(m+2)}\left(\frac{2}{\ln n}\right)^{m / 2} .
$$

Из оценок (28), (29), (31) и формулы (8) получаем (11). Ряд в (11) сходится, и при $x=o(\sqrt{\ln n})$ является асимптотическим разложением в смысле, указанном в формулировке теоремы 2 , поскольку при $\gamma \rightarrow 1 / 2$ ряд в (30) является асимптотическим разложением. Теорема 2 доказана.

Утверждение следствия 2 получается из (12) при $M=0$. Оценка (13) следует из (12) при $M=1$.

Пусть $\xi_{n}-$ случайная величина, равная числу циклов в подстановке, выбранной случайно равновероятно из множества всех $n$ ! подстановок степени $n$. Известно (см. [6]), что, если $n, N \rightarrow \infty$ так, что

$$
N=\ln n+y \sqrt{\ln n}
$$

и выполнено условие (10) с заменой $x$ на $y$, то

$$
\mathbf{P}\left\{\xi_{n}=N\right\}=\frac{(\ln n)^{N}}{N ! n}\left(1+O\left(\frac{|y|}{\ln n}+\frac{1}{\ln n}\right)\right) .
$$

Известно также (см. [7]), что если $N=\gamma \ln n+o(\ln n)$ при $n \rightarrow \infty$, где $\gamma-$ положительная постоянная, то

$$
\mathbf{P}\left\{\xi_{n}=M\right\}=\frac{(\ln n)^{N}}{N ! n \Gamma(\gamma)}(1+o(1)) .
$$

В [8] показано, что соотношение (34) остается справедливым с заменой остаточного члена $o(1)$ на $O(1 / \ln n)$ если $n, N \rightarrow \infty$ так, что выполнено условие (5).

Теорема 3. При условиях теоремь 1 равномерно относительно $\gamma \in\left[\gamma_{0}, \gamma_{1}\right]$

$$
\mathbf{P}\left\{\xi_{n}=N\right\}=\frac{n^{\psi}}{\Gamma(\gamma) \sqrt{2 \pi \gamma \ln n}}(1+O(1 / \ln n))
$$

где

$$
\psi(\gamma)=\gamma(1-\ln \gamma)-1<0
$$

для $\gamma>0, \gamma \neq 1, \psi(1)=0$.

Доказательство. Оценка (35) следует из (34) с учетом (36) (с вышеописанной заменой) и формулы Стирлинга для $N$ !. Теорема 3 доказана.

Следующее утверждение является обобщением (33). 
Теорема 4. Пусть $n$ и $N$ стремятся к бесконечности так, что выполнены условия (33) и (10) (с указанной заменой). Тогда

$$
\begin{array}{r}
\mathbf{P}\left\{\xi_{n}=N\right\}=\frac{1}{\sqrt{2 \pi \ln n}} \exp \left(-\frac{y^{2}}{2}+\sum_{m=1}^{\infty} \frac{(-1)^{m-1} y^{m+2}}{(m+1)(m+2)}(\ln n)^{-m / 2}\right) \\
\times\left(1+O\left(\frac{|y|}{\sqrt{\ln n}}+\frac{1}{\ln n}\right)\right) .
\end{array}
$$

Ряд в (37) сходится при всех достаточно больших значениях $n$ и является асимптотическим разложением в том смысле, что если при $n \rightarrow \infty$

$$
y=y(n)=o\left((\ln n)^{(M+1) /(2 M+6)}\right),
$$

mo

$$
\begin{aligned}
\mathbf{P}\left\{\xi_{n}=N\right\}=\frac{1}{\sqrt{2 \pi \ln n}} \exp \left(-\frac{y^{2}}{2}\right. & \left.+\sum_{m=1}^{M} \frac{(-1)^{m-1} y^{m+2}}{(m+1)(m+2)}(\ln n)^{-m / 2}\right) \\
& \times\left(1+O\left(\frac{|y|}{\sqrt{\ln n}}+\frac{1}{\ln n}+\frac{|y|^{M+3}}{(\ln n)^{(M+1) / 2}}\right)\right),
\end{aligned}
$$

где $M=0,1, \ldots$

Доказательство теоремы 4 проводится аналогично доказательству теоремы 2 с учетом (35), (36), если заметить, что

$$
\Gamma(\gamma)=\Gamma(1+y / \sqrt{\ln n})=1+O(|y| / \sqrt{\ln n})
$$

И

$$
\begin{aligned}
\psi(\gamma) & =\frac{y}{\sqrt{\ln n}}-\left(1+\frac{y}{\sqrt{\ln n}}\right) \ln \left(1+\frac{y}{\sqrt{\ln n}}\right) \\
& =-\frac{y^{2}}{2 \ln n}+\sum_{m=1}^{\infty} \frac{(-1)^{m-1} y^{m+2}}{(m+1)(m+2)}(\ln n)^{-(m+2) / 2}
\end{aligned}
$$

при $|y|<\sqrt{\ln n}$.

Следствие 4. Если $y=y(n)$ при $n \rightarrow \infty$ меняется так, что

$$
y(n)=o\left((\ln n)^{1 / 6}\right),
$$

mo

$$
\mathbf{P}\left\{\xi_{n}=N\right\}=\frac{1}{\sqrt{2 \pi \ln n}} \exp \left(-y^{2} / 2\right)\left(1+O\left(\frac{\left|y+y^{3}\right|}{\sqrt{\ln n}}+\frac{1}{\ln n}\right)\right) .
$$

Следствие 5. Если $y=y(n)=o\left((\ln n)^{1 / 4}\right)$ при $n \rightarrow \infty$, то

$$
\mathbf{P}\left\{\xi_{n}=N\right\}=\frac{1}{\sqrt{2 \pi \ln n}} \exp \left(-\frac{y^{2}}{2}+\frac{y^{3}}{6 \sqrt{\ln n}}\right)\left(1+O\left(\frac{|y|}{\sqrt{\ln n}}+\frac{1+y^{4}}{\ln n}\right)\right) .
$$


Утверждения следствий 4 и 5 получаются из (38) при $M=0$ и $M=1$ соответственно.

Рассмотрим случайную величину $\theta_{n}$, равную числу блоков в разбиении, выбранном случайно и равновероятно из класса всех разбиений множества $\{1,2, \ldots, n\}$. Пусть $B_{n}-$ число таких разбиений (число Белла), тогда (см. стр. 139 в [9])

$$
\mathbf{P}\left\{\theta_{n}=N\right\}=\frac{\sigma(n, N)}{B_{n}}, \quad N=1,2, \ldots, n .
$$

При этом

$$
\sum_{N=1}^{n} \sigma(n, N)=B_{n}, \quad n=1,2, \ldots,
$$

и $\sigma(n, N)$ - числа Стирлинга второго рода.

Известно [10], что при $n \rightarrow \infty$

$$
B_{n}=\frac{1}{\sqrt{\ln n}} \exp (n(r+1 / r-1)-1)(1+o(1)),
$$

где $r$ - единственный положительный корень уравнения

$$
r e^{r}=n,
$$

$r \equiv \ln n$ при $n \rightarrow \infty$, точнее (см. [10]),

$$
r=\ln n-\ln \ln n+\frac{\ln \ln n}{\ln n}+o\left(\frac{\ln \ln n}{\ln n}\right) .
$$

Известно также (см. стр. 141 в [9]), что при $n \rightarrow \infty$

$$
\begin{aligned}
\mathbf{E} \theta_{n} & =\frac{n}{\ln n}(1+o(1)), \\
\mathbf{D} \theta_{n} & =\frac{n}{(\ln n)^{2}}
\end{aligned}
$$

и случайная величина

$$
\frac{\ln n}{\sqrt{n}}\left(\theta_{n}-\mathbf{E} \theta_{n}\right)
$$

имеет в пределе стандартное нормальное распределение.

Если $N=N(n)=o(1)$ при $n \rightarrow \infty$, то (см. [11])

$$
\sigma(n, N)=\frac{N^{n}}{N !} \exp \left(-N e^{-n / N}(1+o(1))\right.
$$

Теорема 5. Пусть $n, N \rightarrow \infty$ так, что

$$
N=\frac{n+z \sqrt{n}}{r}
$$

где

$$
z=z(n)=o(\sqrt{n} / \ln n)
$$


u r-единственный положительный корень уравнения (41). Тогда

$$
\begin{aligned}
\mathbf{P}\left\{\theta_{n}=\right. & M\}=\frac{\ln n}{\sqrt{2 \pi n}} \\
& \times \exp \left(-\frac{z^{2}}{2}\left(1+\frac{1}{r}\right)+\sum_{m=1}^{\infty} \frac{(-1)^{m-1} z^{m+2}}{(m+2) n^{m / 2}}\left(1+\frac{1}{r(m+1)}\right)\right)(1+o(1)),
\end{aligned}
$$

Разложение (45) является асимптотическим разложением в том смысле, что если $z=o\left(n^{(M+1) /(2 M+6)}\right.$ при $n \rightarrow \infty$, то в соответствующем сходящемся ряде достаточно ограничиться первыми М членами.

Доказательство. При условиях (41), (43), (44)

$$
N \equiv \frac{n}{r} \equiv \frac{n}{\ln n},
$$

и поэтому $N=o(n)$, так что справедлива оценка (42).

Кроме того, при этих условиях

$$
N e^{-n / N} \equiv \exp \left(\frac{r z}{\sqrt{n}+z}\right)=1+o(1)
$$

то есть, согласно (42),

$$
\sigma(n, N)=\frac{N^{n}}{N !} e^{-1}(1+o(1)) .
$$

Подставляя эту оценку в (39) и используя (40), получаем, что

$$
\mathbf{P}\left\{\theta_{n}=N\right\}=\frac{N^{n}}{N !} \sqrt{\ln n} \exp (-n(r+1 / r-1)(1+o(1)) .
$$

Формула Стирлинга для $N$ ! с учетом (41), (43), (44), (46), дает равенство

$$
\begin{aligned}
\ln \mathbf{P}\left\{\theta_{n}=N\right\}= & -z \sqrt{n}+\frac{z \sqrt{n}}{r} \\
& +\left(n-\frac{n}{r}-\frac{z \sqrt{n}}{r}\right) \ln (1+z / \sqrt{n})+\ln \left(\frac{\ln n}{\sqrt{2 \pi n}}\right)+o(1) .
\end{aligned}
$$

Поскольку $|z|<\sqrt{n}$ при всех достаточно больших значениях $n$, при таких $n$

$$
\ln (1+z / \sqrt{n})=\sum_{m=1}^{\infty} \frac{(-1)^{m-1} z^{m}}{m n^{m / 2}} .
$$

Подставляя (48) в (47), после элементарных преобразований получаем, что

$$
\ln \mathbf{P}\left\{\theta_{n}=N\right\}=\ln \left(\frac{\ln n}{\sqrt{2 \pi n}}\right)+\sum_{m=0}^{\infty} \frac{(-1)^{m-1} z^{m+2}(m+1+1 / r)}{(m+1)(m+2) n^{m / 2}}+o(1) .
$$

Формула (49) равносильна (45). Из (44) следует, что ряд в (45) является асимптотическим разложением. Теорема 5 доказана.

Следствие 6. При условиях теоремь 5 пусть $z=o(\sqrt{\ln n})$. Тогда

$$
\mathbf{P}\left\{\theta_{n}=N\right\}=\frac{\ln n}{\sqrt{2 \pi n}} \exp \left(-z^{2} / 2\right)(1+o(1)) .
$$




\section{Список литературы}

1. Тимашёв А. Н., Случайные отображения конечных множеств с известным числом компонент. Теория вероятностей и ее применения (2003) 48, №4, 818-828.

2. Колчин В. Ф., Случайные отображения. Наука, Москва, 1984.

3. Harris B., Probability distributions related to random mappings. Ann. Math. Statist. (1960) 31, №4, 1045-1062.

4. Павлов Ю. Л., Предельные распределения одной характеристики случайного отображения. Теория вероятностей и ее применения (1981) 27, №4, 829-834.

5. Чеплюкова И. А., Об одной характеристике случайного отображения с известным числом циклов. Дискретная математика (2006) 18, №3, 43-60.

6. Волынец Л. М., Оценка скорости сходимости к предельному распределению для числа циклов в случайной подстановке. В сб.: Вероятностные задачи дискретной математики. МИЭМ, Москва, 1987, с. 40-46.

7. Колчин В. Ф., Случайные графыл. Наука, Москва, 2000.

8. Тимашёв А. Н., Об асимптотических разложениях для распределения числа циклов в случайной подстановке. Дискретная математика (2003) 15, №3, 117-127.

9. Сачков В. Н., Вероятностные методы а комбинаторном анализе. Наука, Москва, 1978.

10. Moser L. M., Wyman M., An asymptotic for the Bell numbers. Trans. Royal Soc. Canada (1955) 49, 49-54.

11. Медведев Ю. И., Ивченко Г. И., Асимптотические представления конечных разностей степенной функции в произвольной точке. Теория вероятностей и ее применения (1965) 10, №1, 139-144.

Статья поступила 19.12.2008. 